Int. J. Electrochem. Sci., 15 (2020) 4953 - 4963

\title{
Iridium Surface Treatment by Glow Plasma Treatment and Its Application as Electrocatalyst for Methanol and CO Oxidation
}

\author{
Yao Liang ${ }^{1}$, Hongzhong Cai ${ }^{2}$, Jiushuai Deng ${ }^{3}$, Yiqi Cui ${ }^{3, *}$ \\ ${ }^{1}$ City College, Kunming University of Science and Technology, Kunming 650093, China \\ ${ }^{2}$ State Key Laboratory of Advanced Technologies for Comprehensive Utilization of Platinum Metals, \\ Kunming Insitute of Precious Metals, Kunming 650106, China \\ ${ }^{3}$ Faculty of Land Resource Engineering, Kunming University of Science and Technology, Kunming \\ 650093, China \\ *E-mail: $\underline{\text { kmust_cyq@163.com }}$
}

doi: $10.20964 / 2020.06 .34$

Received: 8 February 2020 / Accepted: 19 March 2020 / Published: 10 May 2020

The regulation of the electrocatalytic activity of an iridium surface using a plasma activation treatment process has been studied experimentally and using first-principles calculations. The microstructures and catalytic properties were investigated using scanning electron microscopy, X-ray diffraction, X-ray photoelectron spectroscopy, cyclic voltammetry and CO-stripping voltammograms. The results show that the plasma activation treatment process can promote the surface energy of iridium particles, thereby increasing their surface activity as a catalyst. Furthermore, the current density of Ir/C-Plasma is 1.46 times higher than that of $\mathrm{Ir} / \mathrm{C}$, indicating that the electrocatalytic activity is significantly increased upon increasing the number of surface-active sites. More importantly, the mechanism of the surface activity sites that improves the catalyst performance of the iridium surface was illustrated using first-principle calculations. We also find that $\mathrm{CH}_{3} \mathrm{OH}$ molecules form chemical bonds with the dangling bonds localized on the iridium atoms around the vacancies and that these sites have the lowest adsorption energy $(-0.578 \mathrm{eV})$. Meanwhile, the TS results indicate that the dissociation of $\mathrm{CH}_{3} \mathrm{OH}$ on a non-vacancy surface was more difficult than on the one-vacancy surface. In addition, the charge transfer further confirms that the vacancies on the iridium surface can provide a large number of active sites for chemical adsorption/desorption, which can promote $\mathrm{CO}$ anti-poisoning processes. This study not only reveals the $\mathrm{CH}_{3} \mathrm{OH}$ adsorption/desorption reaction mechanism of Ir/C-Plasma, but also provides effective guidance toward improving the catalyst performance of noble metal catalysts.

Keywords: Electrocatalytic activity, iridium surface, first-principles calculations.

\section{$\underline{\text { FULL TEXT }}$}


(C) 2020 The Authors. Published by ESG (www.electrochemsci.org). This article is an open access article distributed under the terms and conditions of the Creative Commons Attribution license (http://creativecommons.org/licenses/by/4.0/). 